\title{
Abdominal pain in long distance runners: case report and analysis of the literature
}

\author{
F C Dimeo, J Peters, H Guderian
}

Br J Sports Med 2004;38:e24 (http://www.bjsportmed.com/cgi/content/full/38/5/e24)

\begin{abstract}
Abdominal pain is a common complaint among participants in endurance sports. It may be severe, recurrent, and resistant to treatment. There is no direct evidence of the cause of this phenomenon. This report is of a long distance runner who had severe pain in the upper right abdominal quadrant during strenuous exertion. The symptom had been present for several years and did not respond to conservative treatment. Laparoscopy showed congenital supernumerary ligaments binding the gallbladder to the abdominal wall. The complaint resolved after cholecystectomy and resection of adhesions. There was evidence of chronic cholecystitis on histopathological examination. Two years after the operation, he remains free of symptoms. The differential diagnosis of abdominal pain in athletes is discussed.
\end{abstract}

A bdominal pain is a common complaint among participants in endurance sports. Surveys have shown that about one third of runners experience abdominal pain during intense exertion. ${ }^{12}$ More than half a century ago, Herxheimer suggested that the symptom could be caused by the viscera tugging on peritoneal ligaments. ${ }^{3}$ This theory was based on the fact that side pain often occurred during activities that involved frequent jolting movements such as camel riding or driving over rough terrain. Later, it was assumed that the pain was caused by visceral ligament strain at the site of attachment to the diaphragm. However, fluoroscopic examination after a large meal in subjects who experienced abdominal pain while running yielded no evidence of defects in diaphragmatic movement. ${ }^{4}$

More recently, several other factors have been identified or proposed as the cause of abdominal pain during exercise. The most frequently mentioned are mechanical trauma with injury of the intestinal mucosa, hypertrophy of the psoas muscle with compression of the gastrointestinal tract, bloating, hollow viscera spasm, abdominal wall cramps, intrapelvic muscle strain, caecal slap syndrome (repeated trauma of the caecum against a hypertrophied muscular wall), ulceration of the gastric and duodenal mucosa caused by ingestion of non-steroidal anti-inflammatory drugs, motility disturbances resulting from a diet rich in carbohydrate and fibre, extreme mobility of a kidney, irritation of the parietal peritoneum, and an exertion related reduction in the washout of substances with an osmotic effect in the intestinal interstitial fluid. ${ }^{15-13}$ Shoulder tip pain associated with abdominal pain during effort may suggest diaphragmatic irritation. ${ }^{13}$

Abdominal pain is more common in young or inexperienced athletes. ${ }^{214}$ This observation suggests that psychic factors may play a role in the genesis of the problem. In fact, many athletes believe that abdominal pain and other symptoms may be aggravated by anxiety and precompetitive psychic stress. $^{15}$
A further theory postulates that abdominal pain is caused by shunting of blood from the splanchnic bed to the limbs during exercise, and several studies have shown a reduction in mesenteric blood flow during exertion. ${ }^{1}$ This may lead to hypoperfusion and ischaemia of the viscera or diaphragm and generate pain. This is one of the most accepted aetiological theories for exercise related abdominal pain. However, ischaemia does not explain many of the clinical findings. Furthermore, the results of a recent study suggest a lack of correlation between the reduction in abdominal blood flow and pain during effort. ${ }^{16}$

The pounding effect of intestinal jarring has also been considered a direct cause for the development of gastrointestinal symptoms during running. Mechanical stimulation of the intestinal mucosa during running may result in a release of vasoactive intestinal peptide (VIP) $)^{17}$ and prostaglandins, ${ }^{9}$ which promote colonic contraction and could cause abdominal cramps and secretory diarrhoea. Finally, the higher prevalence of abdominal pain in runners than in participants in other endurance sports suggests that mechanical strain on the viscera plays a role in the genesis of this disorder. In fact, body acceleration and deceleration is almost twice as great during running than during cycling, ${ }^{19}$ and abdominal complaints are more common during the running than during the swimming and cycling parts of a triathlon. ${ }^{20}$ The results of several studies show that intense exercise causes substantial stress to the digestive system. After long exertion, occult blood has been detected in the faeces of up to $87 \%$ of participants in endurance events. ${ }^{21}$ In a recent study, endoscopic examination revealed upper gastrointestinal lesions in 15 of 16 runners after a $20 \mathrm{~km}$ race. $^{22}$

Generally, abdominal pain resolves when the effort is stopped or when techniques such as bending forward while tightening the abdominal muscles or breathing through pursed lips after a deep inhalation are applied. ${ }^{16}$ However, abdominal pain is a substantial source of discomfort during athletic events. Moreover, in some cases this problem can be persistent and frequent, or may not respond to treatment. Finally, the uncertainty associated with pain that appears unexpectedly and cannot be successfully treated may erode the self confidence of the athlete and seriously affect athletic performance. A stitch at the wrong moment may make the difference between winning and losing.

We present the case of an athlete who had severe and recurrent abdominal pain during physical exertion.

\section{CASE REPORT}

The patient, a 29 year old national class long distance runner, presented with repeated episodes of abdominal pain during physical exertion. The patient described the pain as severe and stitching; it was localised in the upper right abdominal quadrant and did not radiate to the back, arm, or abdomen. The pain was not accompanied by nausea, vomiting, chills, diarrhoea, or other symptoms. It appeared exclusively during strenuous activities like racing or intense interval training 
sessions and persisted for several minutes after the exertion. Manoeuvres used to reduce the severity of stitch (contraction or relaxation of the abdominal muscles, leaning forward while running, pushing in on the side, or modified breathing) did not alleviate the complaint. The day after a race, the patient was usually free of pain; however, ingestion of fatty meals on the day after a pain attack resulted in mild discomfort in the upper right abdominal quadrant.

The problem had been present for about 12 years. The severity of symptoms and the frequency of pain episodes had increased progressively in the previous few months. In the weeks before consultation, the patient started to experience postprandial discomfort in the upper right abdominal quadrant after fatty meals. He also mentioned that stretching exercises for the abdominal muscles (raising the upper body by extending the arms and keeping the pelvis in floor contact while lying face down) could trigger a pain episode.

The patient had had asthma as a child. He reported two episodes of gastritis five and two years before the consultation. He had no history of surgery, chronic disease, accidents, or hospital admissions and was taking no medicines. He did not consume alcohol regularly. His parents and siblings were healthy.

He had consulted several physicians since the start of his symptoms. To prevent pain attacks, he had been advised to reduce the intake of dietary fat and cellulose, avoid meals for four hours before training or races, and to distend the abdominal muscles and use abdominal breathing exercises during exertion. He had also been taught relaxation techniques. However, none of these measures consistently helped to prevent the pain episodes. He had been prescribed ranitidine, magnesium, pirenzepine, glyceryl trinitrate, and benzodiazepines to prevent pain episodes; none of these drugs yielded positive or long lasting results.

On physical examination, his abdomen was soft and deep palpation did not elicit pain. Liver and spleen were not palpable. Peristaltic sounds were present. All hernial orifices were closed. ECG, stress ECG, blood count, blood sedimentation rate, plasma electrolytes, and tests for hepatic and pancreatic function were normal. An echocardiogram showed a globally and proportionally enlarged heart with a normal ejection fraction and normal contractility. The heart walls were not hypertrophic. Testing for occult blood in the faeces was negative. Abdominal ultrasonography showed local thickening of the cystic wall at the junction between the gallbladder and the cystic duct; no stones, sludge, enlargement of the bladder, or further anatomical deformities were observed.

Owing to the progressive increase in symptom intensity and duration, and as the patient reported new postprandial complaints, a laparoscopy was carried out (fig 1). No hernia or malformations of the abdominal organs were observed. Direct exploration of the abdominal cavity revealed congenital adhesions connecting the gallbladder and liver to the abdominal wall. The surface of the gallbladder was rough and unpolished and its wall was slightly thickened. Lifting the gallbladder from its bed revealed further adhesions between gallbladder, transverse colon and liver. Intraoperative cholangiography showed an unobstructed biliary system and no stones or sludge.

The adhesions were electrocoagulated and sectioned. After cutting the cystic duct, the gallbladder was removed from the abdominal cavity. Pathoanatomical examination showed a tightened and scarred wall with signs of chronic inflammation. Histological examination revealed chronic cholecystitis with inflammatory infiltrates.

The patient had no postoperative complications. He resumed oral food intake after 24 hours and training two weeks after the operation. In the two years after surgery, he

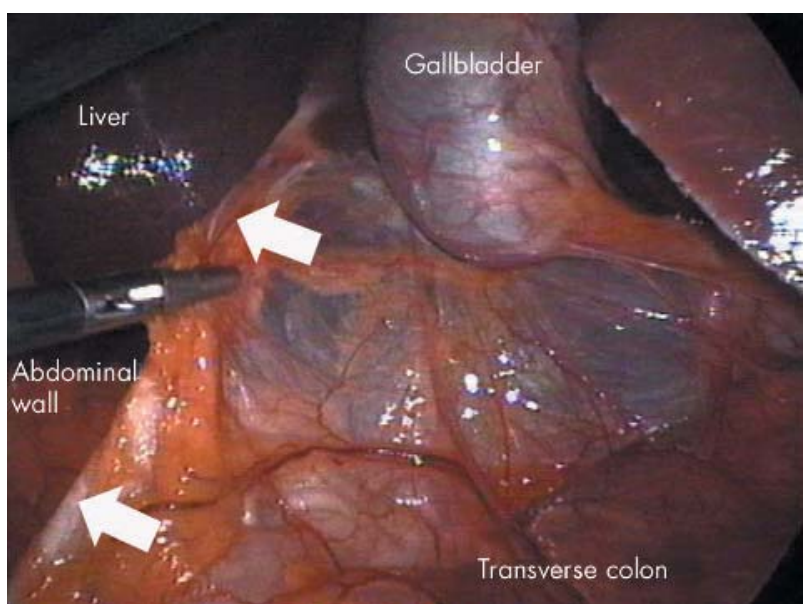

Figure 1 Laparoscopic view of the liver, gallbladder, and transverse colon. Arrow shows supernumerary ligaments connecting colon, abdominal wall, and gallbladder.

has participated in several races and has been free of symptoms.

\section{DISCUSSION}

Abdominal pain during strenuous physical exertion usually has a functional cause and is not related to an underlying disorder. However, in some cases it may be a manifestation of or triggered by diseases such as oesophagitis, gastritis, gastroduodenal ulcer, anomalies of the gallbladder and the biliary system, chronic inflammatory bowel disease, congenital or postoperative intra-abdominal adhesions, nephrolithiasis, or infection. Other causes of abdominal pain during exertion-for example, myocardial ischaemia or atherosclerotic mesenteric angina-are uncommon in athletes.

The aetiology of abdominal pain during exercise is often difficult to determine as the complaints are only present during exertion, and between pain episodes the patients have no signs of disease. Usually the symptoms are self limiting and only require symptomatic treatment. However, they may be frustrating for competitive athletes and they may prevent them from achieving optimal performance during competitions. Furthermore, some circumstances suggest that the cause of pain is not functional. New complaints during exertion in an experienced and previously asymptomatic athlete, the absence of triggering factors such as trauma or dietary excesses, a change in the pattern, duration, or severity of pain, or the persistence of symptoms after exertion all suggest an underlying disorder.

In such cases, basic diagnostic work up should include physical examination, blood count, measurement of hepatic and pancreatic enzymes, and abdominal ultrasonography. A history of postprandial complaints, heartburn, or recurrent pain episodes associated with a positive faecal blood test should be pursued by gastrointestinal endoscopy. Computer scans may help to rule out less common causes of abdominal pain such as neural compression, bone and spine disorders, and intra-abdominal masses. A recent report suggests that gastric tonometry may help diagnosing and grading gastrointestinal ischaemia during exercise. ${ }^{23}$

Explorative laparoscopy is only justified when all the causes of abdominal pain mentioned above have been ruled out. Like any other invasive diagnostic method, laparoscopy can have complications. Thus we feel that it should only be considered when the discomfort is severe or incapacitating, when symptoms also appear during rest, when underlying 


\section{Take home message}

- Abdominal pain during exercise is a common problem but its aetiology is generally unknown. This report describes an athlete who had severe abdominal pain during effort. His complaints resolved after cholecystectomy and laparoscopic resection of congenital supernumerary intra-abdominal ligaments. The findings suggest that exercise related abdominal pain may be caused by visceral tugging during effort.

disease is suspected, and when other diagnostic procedures have failed to explain the cause of the symptoms.

The results of a recent study suggest that mechanical traction on abdominal organs from increased wall tension may be a cause of abdominal pain during physical effort. ${ }^{16}$ Several factors indicate that this mechanism was also the cause of pain in our patient. First, subcostal pain could be reproduced by forced extension of the abdominal muscles. Second, histological examination of the gallbladder showed chronic cholecystitis. This finding tacitly implies that several episodes of acute inflammation had taken place. However, acute inflammation of the gallbladder always generates at least abdominal discomfort. Our patient experienced these symptoms only during exertion, which strongly suggests a causal role for gallbladder irritation in his abdominal pain. However, no biliary stones or sludge, or signs of infection were observed after resection of the biliary ducts. Furthermore, pathoanatomical and laboratory tests ruled out other causes of cholecystitis (vasculitis, obstructive tumour, diabetes mellitus, parasitic or bacterial infection including tuberculosis, or sarcoidosis). Thus repeated mechanical tugging is the most likely cause of acute cholecystitis in this otherwise healthy young man. Third, and most importantly, the pain episodes resolved after cholecystectomy.

The finding of chronic cholecystitis in our patient shows that stitch is not always an irrelevant symptom. While occasional side pain may be caused by a functional disorder and not require further treatment, persistent or increasing complaints should prompt the clinician to carry out a thorough diagnostic evaluation of the problem.

\section{Authors' affiliations}

F C Dimeo, Department of Haemotology, Oncology and Transfusion Medicine, Charité Universitätsmedizin Berlin, Campus Benjamin Franklin, Hindenburgdamm, Berlin, Germany
J Peters, Department of Gastroenterology, Infectology and

Rheumatology, Charité Universitätsmedizin Berlin

H Guderian, Stadtstrasse 72, 73104, Freiburg, Germany

Correspondence to: Dr Fernando Carlos Dimeo, Charité

Universitätsmedizin Berlin, Campus Benjamin Franklin, Station 06, Hindenburgdamm 30, Berlin 12200, Germany; fernando.dimeo@ charite.de

Accepted 29 August 2003

\section{REFERENCES}

1 Gil SM, Yazaki E, Evans DF. Aetiology of running-related gastrointestinal dysfunction. How far is the finishing line? Sports Med 1998:26:365-78.

2 Riddoch C, Trinick T. Gastrointestinal disturbances in marathon runners. Br J Sports Med 1988;22:71-4.

3 Herxheimer H. On the side stitsch (Über das "Seitenstechen"). Arch Intern Med 1941;68:94-101.

4 Sinclair JD. Stitch: the side pain of athletes. NZ Med J 1951;50:607-12.

5 Porter AM. Marathon running and the caecal slap syndrome. Br J Sports Med 1982;16:178

6 Porter AM. Non-steroidal anti-inflammatory drugs and ethanol. Br J Sports Med 1982;16:265.

7 Dawson M. Psoas muscle hypertrophy: mechanical cause for jogger's trots. BMJ 1985;291:787-8.

8 Granger DN, Richardson PD, Kvietys PR, et al. Intestinal blood flow. Gastroenterology 1980;78:837-63.

9 Beubler E, Juan H. PGE-release, blood flow and transmucosal water movement after mechanical stimulation of the rat jejunal mucosa. Naunyn Schmiedebergs Arch Pharmacol 1978;305:91-5

10 Gallavan RH, Jacobson, eds. Prostaglandins and the splanchnic circulation. Proc Soc Exp Biol Med 1982;170:391-7.

11 Green GA. Gastrointestinal disorders in the athlete. Clin Sports Med 1992;11:453-70.

12 Leslie BR. Exercise-induced abdominal pain. JAMA 1983;250:3283.

13 Morton DP, Callister R. Characteristics and etiology of exercise-related transient abdominal pain. Med Sci Sports Exerc 2000:32:432-8.

14 Morton DP, Callister R. Factors influencing exercise-related transient abdominal pain. Med Sci Sports Exerc 2002;34:745-9.

15 Priebe W, Priebe J. Runner's diarrhoea - prevalence and clinical symptomatology. Am J Gastroenterol 1988;79:827-8.

16 Plunkett BT, Hopkins WG. Investigation of the side pain "stitch" induced by running after fluid ingestion. Med Sci Sports Exerc 1999;31:1169-75.

17 Opstad PK. The plasma vasoactive intestinal peptide (VIP) response to exercise is increased after prolonged strain, sleep and energy deficiency and extinguished by glucose infusion. Peptides 1987; 8:175-8.

18 MacLaren DP, Raine NM, O'Connor AM, et al. Human gastrin and vasoactive intestinal polypeptide responses to endurance running in relation to training status and fluid ingested. Clin Sci (Lond) 1995;89:137-43.

19 Rehrer NJ, Meijer GA. Biomechanical vibration of the abdominal region during running and bicycling. J Sports Med Phys Fitness 1991;31:231-4.

20 Sullivan SN, Wong C. Runners' diarrhea. Different patterns and associated factors. J Clin Gastroenterol 1992;14:101-4.

21 Rudzki SJ, Hazard H, Collinson D. Gastrointestinal blood loss in triathletes: it's etiology and relationship to sports anaemia. Aust J Sci Med Sport 1995:27:3-8.

22 Choi SC, Choi SJ, Kim JA, et al. The role of gastrointestinal endoscopy in longdistance runners with gastrointestinal symptoms. Eur J Gastroenterol Hepatol $2001 ; 13: 1089-94$

23 Kolkman JJ, Groeneveld AB, van der Berg FG, et al. Increased gastric PCO2 during exercise is indicative of gastric ischaemia: a tonometric study. Gut 1999;44: 163-7. 